\title{
Optimization of Improved Motion-sensitized Driven-equilibrium (iMSDE) blood suppression for carotid artery wall imaging
}

Chengcheng Zhu ${ }^{1 *}$, Martin J Graves ${ }^{1}$, Jianmin Yuan ${ }^{1}$, Umar Sadat ${ }^{2}$, Jonathan H Gillard ${ }^{1}$ and Andrew J Patterson ${ }^{1}$

\begin{abstract}
Background: Improved motion-sensitized driven-equilibrium (iMSDE) preparations have been successfully used in carotid artery wall imaging to achieve blood suppression, but it causes notable signal loss, mostly due to inherent $\mathrm{T}_{2}$ decay, eddy current effects and $\mathrm{B}_{1}^{+}$inhomogeneity. In this study, we investigate the signal to noise ratio (SNR) and blood suppression performance of iMSDE using composite RF pulses and sinusoidal gradients. Optimized first moment $\left(m_{1}\right)$ values for iMSDE prepared $T_{1}$ - and $T_{2}$ - weighted $\left(T_{1}\right.$ - and $\left.T_{2}-w\right)$ imaging are presented.

Methods: Twelve healthy volunteers and six patients with carotid artery disease underwent IMSDE and double inversion recovery (DIR) prepared $T_{1}$ - and $T_{2}-W$ fast spin echo (FSE) MRI of the carotid arteries. Modified iMSDE module using composite RF pulses and sinusoidal gradients were evaluated with a range of $\mathrm{m}_{1}$. SNR of adjacent muscle, vessel wall and the lumen were reported. The optimized iMSDE module was also tested in a 3D variable flip angle FSE (CUBE) acquisition.

Results: The SNR of muscle was highest using sinusoidal gradients, and the relative improvement over the trapezoidal gradient increased with higher $m_{1}(p<0.001)$. Optimal SNR was observed using an iMSDE preparation scheme containing two $180^{\circ}$ composite pulses and standard $90^{\circ}$ and $-90^{\circ}$ pulses $(p=0.151$ ). iMSDE produced better blood suppression relative to DIR preparations even with a small $m_{1}$ of $487 \mathrm{mT}^{*} \mathrm{~ms}^{2} / \mathrm{m}(\mathrm{p}<0.001)$. In $\mathrm{T}_{1}-\mathrm{W}$ iMSDE, there was a SNR decrease and an increased $T_{2}$ weighting with increasing $m_{1}$. In $T_{2}-w$ iMSDE, by matching the effective echo time (TE), the SNR was equivalent when $\mathrm{m}_{1}$ was $<=1518 \mathrm{mT}^{*} \mathrm{~ms}^{2} / \mathrm{m}$, however, higher $\mathrm{m}_{1}$ values $\left(2278-3108 \mathrm{mT}^{*} \mathrm{~ms}^{2} / \mathrm{m}\right)$ reduced the SNR. In the patient study, iMSDE improved blood suppression but reduced vessel wall CNR efficiency in both $T_{1}-w$ and $T_{2}-w$ imaging. iMSDE also effectively suppressed residual flow artifacts in the CUBE acquisition.
\end{abstract}

Conclusions: iMSDE preparation achieved better blood suppression than DIR preparation with reduced vessel wall CNR efficiency in $T_{1}-w$ and $T_{2}-w$ images. The optimized $m_{1} s$ are $487 \mathrm{mT}^{*} \mathrm{~ms}^{2} / \mathrm{m}$ for $\mathrm{T}_{1}$-w imaging and 1518 $\mathrm{mT}^{*} \mathrm{~ms}^{2} / \mathrm{m}$ for $\mathrm{T}_{2}-\mathrm{W}$ imaging. Composite $180^{\circ}$ refocusing pulses and sinusoidal gradients improve SNR performance. iMSDE further improves the inherent blood suppression of CUBE.

Keywords: Carotid atheroma, Blood suppression optimization, Fast spin echo, Motion-sensitized driven-equilibrium

\footnotetext{
* Correspondence: zhucheng043@gmail.com

${ }^{1}$ University Department of Radiology, University of Cambridge, Cambridge CB2 OQQ, UK

Full list of author information is available at the end of the article
}

\section{Biomed Central}

(c) 2014 Zhu et al.; licensee BioMed Central Ltd. This is an Open Access article distributed under the terms of the Creative Commons Attribution License (http://creativecommons.org/licenses/by/2.0), which permits unrestricted use, distribution, and reproduction in any medium, provided the original work is properly credited. 


\section{Background}

Carotid atheroma is a known risk factor for subsequent stroke [1]. High resolution cardiovascular magnetic resonance (CMR) of the carotid artery allows for the visualisation and quantification of plaque composition and morphology and helps patient risk stratification [2]. Blood suppression is essential for black blood vessel wall imaging, and unsuppressed intra-luminal blood leads to plaque mimicking artifacts [3]. Double inversionrecovery (DIR) methods [4] have become the de facto standard for blood suppressed vessel wall imaging. However, this method is sensitive to the rate of flow replenishment which leads to plaque-mimicking artefacts in the presence of slow and turbulent flow that often occurs around the carotid bifurcation [3].

Motion-sensitized driven-equilibrium (MSDE) preparation has been proposed as an alternative blood suppression technique [5,6]. An improved version of MSDE (iMSDE) has also been proposed which demonstrated better SNR performance $[7,8]$. The blood suppression capability of iMSDE is not limited by the rate of flow replenishment, and it can suppress blood flow in every direction by applying flow sensitizing gradients along each axis. iMSDE has the potential to suppress turbulent and slow blood flow, which requires the prescription of higher $\mathrm{m}_{1}$ values. Furthermore, since iMSDE requires a shorter preparation time relative to DIR preparation, it is more time efficient.

One limitation of iMSDE preparation is that it causes notable signal loss, mostly due to $\mathrm{T}_{2}$ decay during the iMSDE preparation time. It is also sensitive to eddy current effects and $B_{1}^{+}$non-uniformity [8]. Signal loss also becomes worse when high $\mathrm{m}_{1}$ values are prescribed [6].

$\mathrm{T}_{1}$ - and $\mathrm{T}_{2}$-weighted $\left(\mathrm{T}_{1^{-}}\right.$and $\mathrm{T}_{2}$-w) double inversion recovery (DIR) prepared fast spin echo (FSE) sequences have been extensively used for plaque component characterization [2], however, carotid wall CMR with iMSDE preparation were rarely reported. Wang et al previously reported iMSDE prepared proton-densityweighted (PD-w) with $\mathrm{m}_{1}$ of 945 and $1524 \mathrm{mT}^{* *} \mathrm{~ms}^{2} / \mathrm{m}$ [8]. Balu et al previously reported an iMSDE prepared 3D MERGE sequence with an $\mathrm{m}_{1}$ of $1524 \mathrm{mT}^{*} \mathrm{~ms}^{2} / \mathrm{m}$ [7]. However, to date there is a paucity of data comparing DIR and iMSDE to achieve optimal blood suppression and a lack of data on optimised iMSDE preparation timings for $\mathrm{T}_{1}$ - and $\mathrm{T}_{2}$-w FSE protocols.

This study measures iMSDE induced signal intensity decay through phantom and volunteer studies. The optimized techniques were then tested on patients. These experiments were conducted across a range of first order moments $\left(\mathrm{m}_{1}\right)$ used in the literature [8] and across $\mathrm{T}_{2}$ values known to reflect plaque composition [9]. Composite radiofrequency $(\mathrm{RF})$ pulses are known to potentially reduce the signal loss due to $B_{1}^{+}$non-uniformity [10], and sinusoidal gradients can potentially reduce eddy current effects. In this study, we report optimizations of SNR and blood suppression performance in $\mathrm{iMSDE}$ prepared carotid vessel wall CMR using composite RF pulses and sinusoidal gradients. DIR and iMSDE prepared $\mathrm{T}_{1}$ and $\mathrm{T}_{2}-\mathrm{w}$ protocols are then presented and compared in a group of normal volunteers and patients.

3D black blood variable flip angle FSE sequences are currently topical and have been applied to carotid imaging due to their intrinsic blood suppression ability and high SNR efficiency [11] [12,13]. However, residual artefacts can still appear at the carotid bifurcation, due to slow and complex flow [14]. In this study we briefly evaluate if the optimized iMSDE module can suppress these artefacts.

\section{Methods}

\section{Study design}

The experimental observations reported in this study were conducted on uniform test objects, calibrated gel phantoms, normal volunteers and asymptomatic patients. Both the volunteer and patient experiments were conducted following local ethical committee approval, all subjects involved gave informed written consent. The institutional review board of Addenbrooke's hospital (Cambridge, UK) approved the whole study. The normal volunteers had the following demographics: $\mathrm{n}=12$, aged 25-50 years, 8 male. The patients had confirmed stenosis $>30 \%$ and the following demographics: $n=6$, mean age 65.7 years [range: 54 to 76 years], male $=3$.

The test object experiments examined the image nonuniformity using permutations of standard and composite RF pulses, and compared theoretical and observed SNR change as a function of $\mathrm{m}_{1}$ using both trapezoidal and sinusoidal gradient types. The volunteer experiments measured sternocleidomastoid (SCM) muscle SNR changes using different RF pulse types, and also measured the intra-lumen and SCM muscle SNR change as a function of $\mathrm{m}_{1}$ and motion sensitizing gradient type. Optimizations for effective preparation regimes are reported for $T_{1}$ - and $\mathrm{T}_{2}$-w protocols by comparing conventional DIR preparations with iMSDE preparations with varying $\mathrm{m}_{1}$. The optimized iMSDE protocol was then validated on patients.

Pulse sequence diagrams of the iMSDE preparations used are shown in Figure 1. The specific parameters used to obtain the reported first order moments are listed in Table 1. The imaging experiments were conducted on a 1.5 T MR system (MR450, GE Healthcare, Waukesha, WI).

\section{Phantom study \\ Composite RF pulses}

A uniform cylindrical test object was scanned using a transmit/receive birdcage head coil. A PDw FSE sequence was prescribed with the following parameters: $\mathrm{TR} / \mathrm{TE}_{\mathrm{eff}}$ : $3000 \mathrm{~ms} / 8.8 \mathrm{~ms} ; 16 \mathrm{~cm} \times 16 \mathrm{~cm}$ field of view (FOV), 


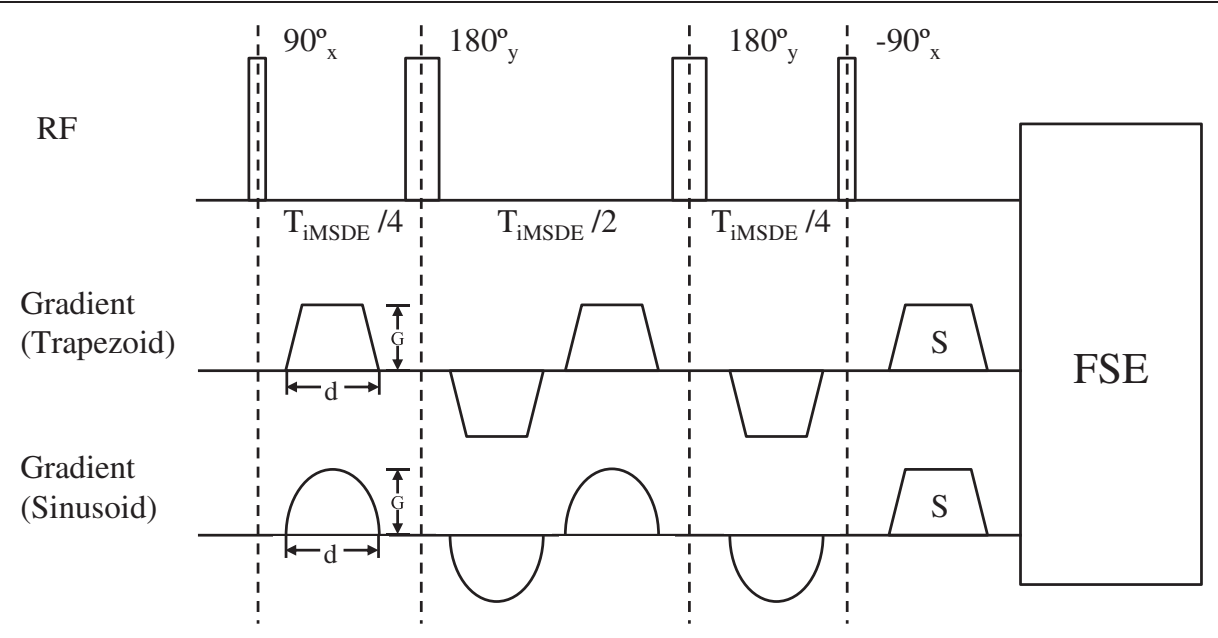

Figure 1 Diagram of iMSDE preparation with trapezoid and sinusoid gradients. Gradient amplitude (G) and duration (d) of the flow sensitive gradient are defined.

$256 \times 256$ matrix, $5 \mathrm{~mm}$ slice thickness; number of excitations $(\mathrm{NEX})=1$; echo train length $(\mathrm{ETL})=10$. A single slice was prescribed through the middle of the phantom. iMSDE preparations were prescribed using variable RF pulse types including: no composite pulse (NOCP), composite $-90^{\circ}$ (CP90), composite $180^{\circ}$ (CP180) and both composite $180^{\circ}$ and $-90^{\circ}$ (CP180_90). Image non-uniformity was examined and compared between sequences. The use of a transmit/receive birdcage head coil allowed us to compare signal intensity variation as a function of the iMSDE preparation regime without dominant spatial location signal intensity variation as a result of RF reception $\left(\mathrm{B}_{1}^{-}\right)$non-uniformity.

\section{Motion sensitizing gradient and first order moment}

Two gels with known $\mathrm{T}_{2}$ values of $50 \mathrm{~ms}$ and $89 \mathrm{~ms}$ respectively (Eurospin Test Object TO5, Diagnostic Sonar Ltd, West Lothian, Scotland, UK) were scanned using the same head coil. The gels were selected to best correspond with the reported $\mathrm{T}_{2}$ values of plaque [9] and SCM tissue $[15,16]$ found in vivo. Since no in vivo
$\mathrm{T}_{2}$ measurement of SCM in $1.5 \mathrm{~T}$ was available, its $\mathrm{T}_{2}$ value was approximated using values from $0.5 \mathrm{~T}$ and $3 \mathrm{~T}[15,16]$.

A PDw FSE sequence was prescribed with the following parameters: TR/TE eff: $3000 \mathrm{~ms} / 8.8 \mathrm{~ms} ; 24 \mathrm{~cm} \times$ $24 \mathrm{~cm}$ FOV, $256 \times 256$ matrix, $5 \mathrm{~mm}$ slice thickness; $\mathrm{NEX}=1 ; \mathrm{ETL}=10$. A single slice was prescribed through the middle of the gels. iMSDE preparations were prescribed using trapezoidal and sinusoidal gradients with $\mathrm{m}_{1}$ values ranging from 181 to $3108 \mathrm{mT}^{*} \mathrm{~ms}^{2} / \mathrm{m}$ (defined in Table 1 ) using a composite $180^{\circ}$ pulse. The experiment was then repeated to acquire a conventional PDw image without iMSDE preparation. The SNR of the gels was measured and compared between sequences.

\section{Theoretical model}

The theoretical model for $\mathrm{T}_{2}$ decay effects during the iMSDE preparation was calculated as $e^{-T_{i M S D E} / T_{2}}$, where $\mathrm{T}_{\mathrm{iMSDE}}$ is the time between the 90 and $-90 \mathrm{RF}$ pulses in the iMSDE module.

Table 1 Parameters for iMSDE using sinusoidal and trapezoidal gradients with increasing first order moment ( $m_{1}$ )

\begin{tabular}{|c|c|c|c|c|}
\hline \multirow{2}{*}{$\begin{array}{l}\mathrm{m}_{1}\left(\mathrm{mTms}^{2} / \mathrm{m}\right) \\
181\end{array}$} & \multicolumn{2}{|c|}{$\begin{array}{l}\text { Gradient strength }(\mathrm{G})(\mathrm{mT} / \mathrm{m}) \\
\text { Trapezoidal/Sinusoidal }\end{array}$} & \multirow{2}{*}{$\begin{array}{l}\text { Gradient duration } \\
(\mathrm{d})^{\mathrm{a}}(\mathrm{ms})\end{array}$} & \multirow{2}{*}{$\begin{array}{l}\begin{array}{l}\text { Duration of preparation } \\
\text { pulse }^{\text {b }}(\mathbf{m s})\end{array} \\
10.0\end{array}$} \\
\hline & 20 & 26 & & \\
\hline 487 & 20 & 28 & 2.0 & 13.6 \\
\hline 925 & 20 & 29 & 2.9 & 17.2 \\
\hline 1518 & 20 & 30 & 3.4 & 21.0 \\
\hline 2278 & 20 & 30 & 4.8 & 24.8 \\
\hline 3108 & 20 & 30 & 5.7 & 28.4 \\
\hline
\end{tabular}

${ }^{\mathrm{a}}$ The duration of one gradient lobe.

${ }^{\mathrm{b}}$ The duration from the start of the first $90^{\circ}$ pulse to the end the $-90^{\circ}$ pulse. 


\section{Volunteer and Patient studies}

Twelve healthy volunteers underwent DIR and iMSDE prepared, ungated, $\mathrm{T}_{1^{-}}$and $\mathrm{T}_{2}-\mathrm{w}$ imaging of the carotid arteries using a 4-channel phased-array carotid coil (PACC, Machnet BV, Elde, The Netherlands). Scan parameters for the FSE sequence were: TR/TE $\left(\mathrm{T}_{1}-\mathrm{w}\right)$ : $800 \mathrm{~ms} / 10 \mathrm{~ms} ; \mathrm{TR} / \mathrm{TE}_{\mathrm{eff}}\left(\mathrm{T}_{2}-\mathrm{w}\right): 2500 \mathrm{~ms} / 50 \mathrm{~ms}$; $14 \mathrm{~cm} \times 14 \mathrm{~cm}$ FOV, $256 \times 192$ matrix, $3 \mathrm{~mm}$ slice thickness, $\mathrm{NEX}=2$; ETL $\left(\mathrm{T}_{1}-\mathrm{w}\right)=10$; ETL $\left(\mathrm{T}_{2}-\mathrm{w}\right)=12$, acquiring 4 slices to cover the bifurcation. A single and multi-slice DIR sequence was prescribed as defined previously [4]. Both iMSDE and DIR sequences acquired 1 slice per $T R$ in the $T_{1}-\mathrm{W}$ protocol and 4 slices per TR for the $T_{2}-w$ protocol. To offset the inherent $T_{2}$ decay during the iMSDE preparation, the preparation time was included in the calculation of the effective TE, i.e. prescribed $\mathrm{TE}=\mathrm{TE}_{\text {eff }}-\mathrm{T}_{\mathrm{iMSDE}}$. For example, in $\mathrm{T}_{2}-\mathrm{w}$ iMSDE acquisition with an $\mathrm{m}_{1}$ of $921 \mathrm{mT}^{*} \mathrm{~ms}^{2} / \mathrm{m}$ and a preparation time $17.2 \mathrm{~ms}$, the prescribed TE was calculated as: $50 \mathrm{~ms}-17.2 \mathrm{~ms}=32.8 \mathrm{~ms}$.

Six volunteers underwent iMSDE prepared $\mathrm{T}_{1}$-w FSE sequences using composite RF pulses and sinusoidal/ trapezoidal gradients. Six separate volunteers also underwent combinations of DIR and iMSDE prepared $\mathrm{T}_{1}$ - and $\mathrm{T}_{2^{-}} \mathrm{w}$ imaging using sinusoidal gradients to determine optimal $\mathrm{m}_{1}$.

For validation purposes six patients were scanned with optimized settings for $\mathrm{T}_{1}$ and $\mathrm{T}_{2}-\mathrm{w}$ protocols.

To evaluate the iMSDE module within the $3 \mathrm{D}$ variable flip angle FSE sequence (CUBE, GE Healthcare, Waukesha, WI), a volunteer was scanned with and without iMSDE preparation with the following scan parameters: TR/TE: $440 \mathrm{~ms} / 10 \mathrm{~ms} ; 14 \mathrm{~cm} \times 14 \mathrm{~cm}$ FOV; $224 \times 224$ matrix; 40 coronal slices; $1.2 \mathrm{~mm}$ slice thickness (interpolated to $0.6 \mathrm{~mm}$ ); ETL 24. A 487 $\mathrm{mT}^{*} \mathrm{~ms}^{2} / \mathrm{m} \mathrm{m}_{1}$ and sinusoidal gradients were used for the iMSDE preparation.

\section{Image analysis}

For each carotid artery, 4 slices were selected for analysis. Measurements of SNR in the adjacent SCM muscle and within the lumen were performed using CMR tools (Cardiovascular Imaging Solutions, London, UK). In the six patients, the vessel wall SNR was also measured. Noise was determined as the standard deviation of the signal in an artefact free background region. SNR was calculated as:

$$
\mathrm{SNR}=0.695 \times \mathrm{S} / \sigma
$$

Where $S$ is the signal intensity, $\sigma$. is the standard deviation of the noise, and the multiplier 0.695 corresponds to a four-channel coil correction [17]
Contrast to noise ratio (CNR) of muscle and wall was defined as:

$$
\begin{array}{r}
\mathrm{CNR}_{\text {eff, muscle }}=\left(\mathrm{SNR}_{\text {muscle }}-\mathrm{SNR}_{\text {lumen }}\right) /\left(\sqrt{\mathrm{T}_{\mathrm{sl}}} \mathrm{SL}_{\text {th }}\right) . \\
([2]) \\
\mathrm{CNR}_{\text {eff, wall }}=\left(\mathrm{SNR}_{\text {wall }}-\mathrm{SNR}_{\text {lumen }}\right) /\left(\sqrt{\mathrm{T}_{\mathrm{sl}}} \mathrm{SL}_{\text {th }}\right) \cdot \quad([3])
\end{array}
$$

Where $\mathrm{T}_{\mathrm{sl}}$. is the scan time (in minutes) per slice, and $\mathrm{SL}_{\mathrm{th}}$. is the slice thickness (in $\mathrm{mm}$ ).

\section{Statistical analysis}

Normality assumptions were formally assessed using a Shapiro-Wilk's test. Distributions were summarised using the median [inter-quartile range]. An ANOVA was performed to test group differences between muscle and luminal SNR and CNR using the different composite RF preparations and also to test differences between sinusoidal and trapezoidal gradients. A Bonferroni correction was applied to account for the multiple comparison problem. Therefore, to account for the fact that 16 formal hypotheses have been investigated statistical significance was defined as $\mathrm{p}<0.003125$ i.e. $0.05 / 16$. SNR and CNR comparison between groups was performed using nonparametric Wilcoxon signed-rank test. All the statistical analyses were performed using the statistical programming language $\mathrm{R}$ version 2.7.0 (The R Foundation of Statistical Computing, Vienna, Austria).

\section{Results}

The test object study demonstrated that a composite $180^{\circ}$ pulse improved the image uniformity, whereas the addition of a composite $-90^{\circ}$ pulse decreased the uniformity (Figure 2). We also observed that signal intensity decreased as a function of increasing first order moment $\mathrm{m}_{1}$ (Figure 3 ). $\mathrm{T}_{2}$ decay during $\mathrm{T}_{\mathrm{iMSDE}}$ was responsible for the majority of the signal decrease. iMSDE using sinusoidal gradients demonstrated a better SNR performance compared to iMSDE using trapezoidal gradients with equivalent $m_{1} s$, with this trend becoming more significant with increasing $\mathrm{m}_{1}$. This reduction was therefore attributed to eddy current effects. In addition, the signal drop was more significant in the gel with the shorter $T_{2}$.

In the volunteer study we observed that the composite $180^{\circ}$ (CP180) yielded the best SNR performance (Figure 4 and Table 2). We noted that adding a composite $-90^{\circ}$ pulse reduced the SNR. Although the CP180 method appeared to provide the highest muscle SNR, we found no statistically significant SNR ( $=0.151)$ or blood suppression $(\mathrm{p}=0.387)$ differences between RF preparations in vivo.

$\mathrm{T}_{1}-\mathrm{w}$ iMSDE preparation using sinusoidal gradients showed better muscle SNR compared with trapezoidal 


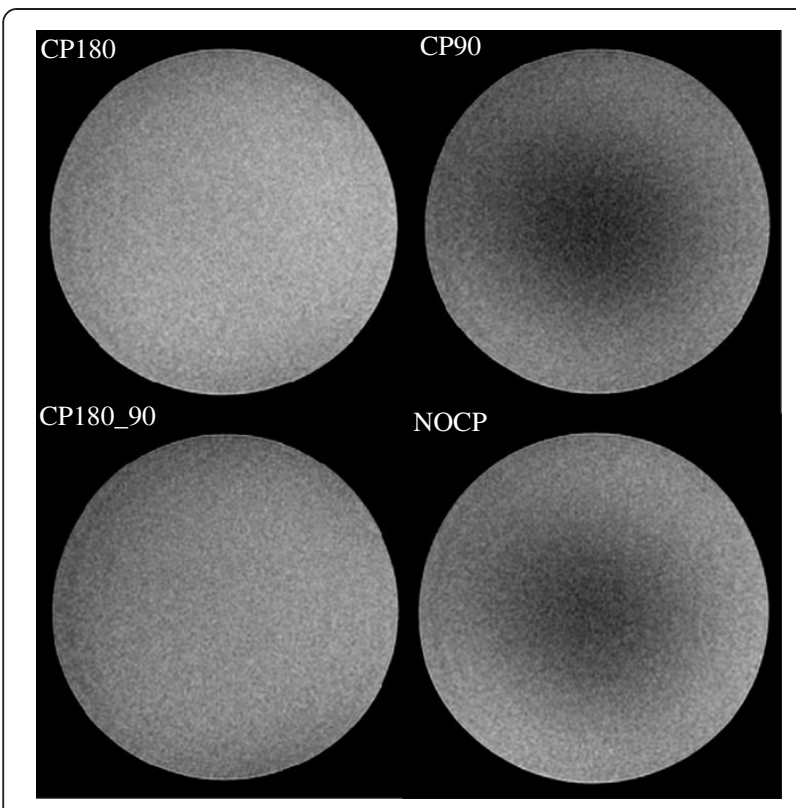

Figure 2 PDw phantom images using varying iMSDE RF pulse types with identical windowing. Composite $180^{\circ}$ only (CP180), composite $-90^{\circ}$ only (CP90), composite $180^{\circ}$ and composite $-90^{\circ}$ (CP180_CP90), and no composite RF pulses (NOCP). Sinusoidal gradients with a first order moment of $487 \mathrm{mT}^{*} \mathrm{~ms}^{2} / \mathrm{m}$ were used.

gradients (Figure 5 and Table 3). This improvement was more significant with increasing $\mathrm{m}_{1}$ (6.5\% higher SNR for $487 \mathrm{~m}_{1}, 10.8 \%$ higher SNR for $1518 \mathrm{~m}_{1}, 16.4 \%$ higher SNR for $3108 \mathrm{~m}_{1}, \mathrm{p}<0.001$ ) (Table 3). There was no significant difference in blood suppression as a function of gradient type $(\mathrm{p}=0.338)$.

$\mathrm{T}_{1}$-w or $\mathrm{T}_{2}$-w iMSDE demonstrated better blood suppression compared with DIR (Figures 6 and 7) even with a small $\mathrm{m}_{1}$ value of $487 \mathrm{mT}^{*} \mathrm{~ms}^{2} / \mathrm{m}(\mathrm{p}<0.001)$. In $\mathrm{T}_{1}$-w iMSDE, the muscle SNR decreased with increasing $m_{1}$.
The lumen SNR decreased with the increased $\mathrm{m}_{1}$, but when the $\mathrm{m}_{1}$ was greater than $925 \mathrm{mT}^{*} \mathrm{~ms}^{2} / \mathrm{m}$, no further improvement was observed. In $\mathrm{T}_{2}-\mathrm{w}$ iMSDE, by keeping the effective TE equivalent, no measurable SNR loss was found when $m_{1}$ less than or equal to $1518 \mathrm{mT}^{* *} \mathrm{~ms}^{2} / \mathrm{m}$. Lumen SNR decreased with increased $\mathrm{m}_{1}$, but the improvement was negligible when $\mathrm{m}_{1}$ was greater than or equal to $1518 \mathrm{mT}^{*} \mathrm{~ms}^{2} / \mathrm{m}$.

The optimized iMSDE prepared $\mathrm{T}_{1} \mathrm{w}$ and $\mathrm{T}_{2} \mathrm{w}$ protocols were applied in six patients. Good image quality was noted with better blood suppression $(\mathrm{p}<0.001)$ with respect to the DIR method (Figure 8 and Table 4). In iMSDE $\mathrm{T}_{1} \mathrm{w}$ imaging, the SNR loss in muscle was $40.3 \%$, whilst the SNR loss in the wall was $34.6 \%$. In iMSDE $\mathrm{T}_{2} \mathrm{w}$ imaging, the SNR of muscle increased by $5.8 \%$ (due to the shorter effective TE) whilst the wall SNR decreased by $22.2 \%$.

The optimized iMSDE module also removed flow artefacts in a $\mathrm{T}_{1} \mathrm{w}$ CUBE sequence but a reduction in image SNR was noted (Figure 9).

\section{Discussion}

DIR prepared FSE sequences have been extensively used for carotid plaque imaging [2]. Wang et al previously reported that MSDE preparation achieved better blood suppression and higher muscle to lumen CNR efficiency than multi-slice DIR when using a $\mathrm{T}_{2}-\mathrm{w}$ FSE sequence [5]. Recently, Obara et al found 3D iMSDE prepared turbo field echo sequences had a higher muscle to lumen CNR efficiency than DIR prepared 2D FSE sequences for both $\mathrm{T}_{1}-\mathrm{w}$ and $\mathrm{T}_{2}-\mathrm{w}$ imaging in a group of five volunteers [18]. However, to date there have been no studies comparing the relative blood suppression and vessel wall CNR performance of iMSDE and DIR prepared FSE in generating the most clinically relevant $\mathrm{T}_{1^{-}}$and $\mathrm{T}_{2^{-}}$contrast
(A)

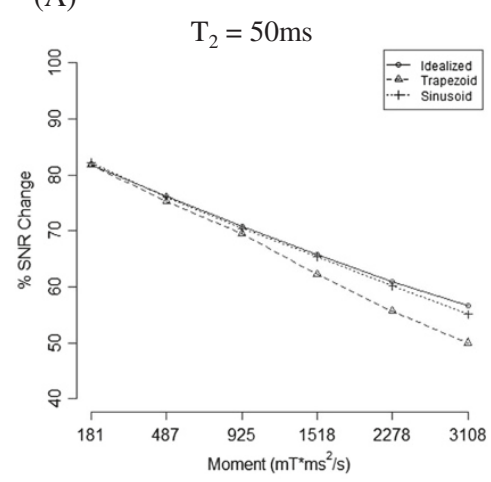

(B)

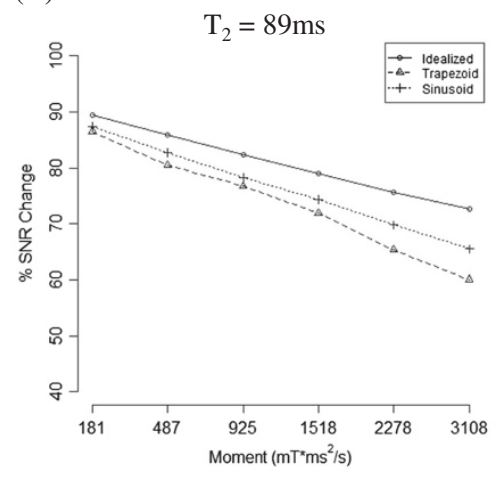

Figure 3 Percentage SNR decrease using iMSDE relative to the standard sequence without blood suppression (in gel phantoms). Comparison of SNR change is plotted against the idealized case (computing theoretical signal intensity change as a function of the increased effective echo time (TEeff)). Experimental observations of signal intensity change using trapezoidal and sinusoidal gradients for motion sensitization are illustrated using two calibrated gel phantoms with T2 values of $50 \mathrm{~ms}$ (A) and $89 \mathrm{~ms}$ (B) $\left[1.5 \mathrm{~T} @ 21^{\circ} \mathrm{C}\right]$ to represent typical carotid vessel wall and muscle tissue respectively. A composite $180^{\circ}$ pulse was used for all iMSDE preparations. 


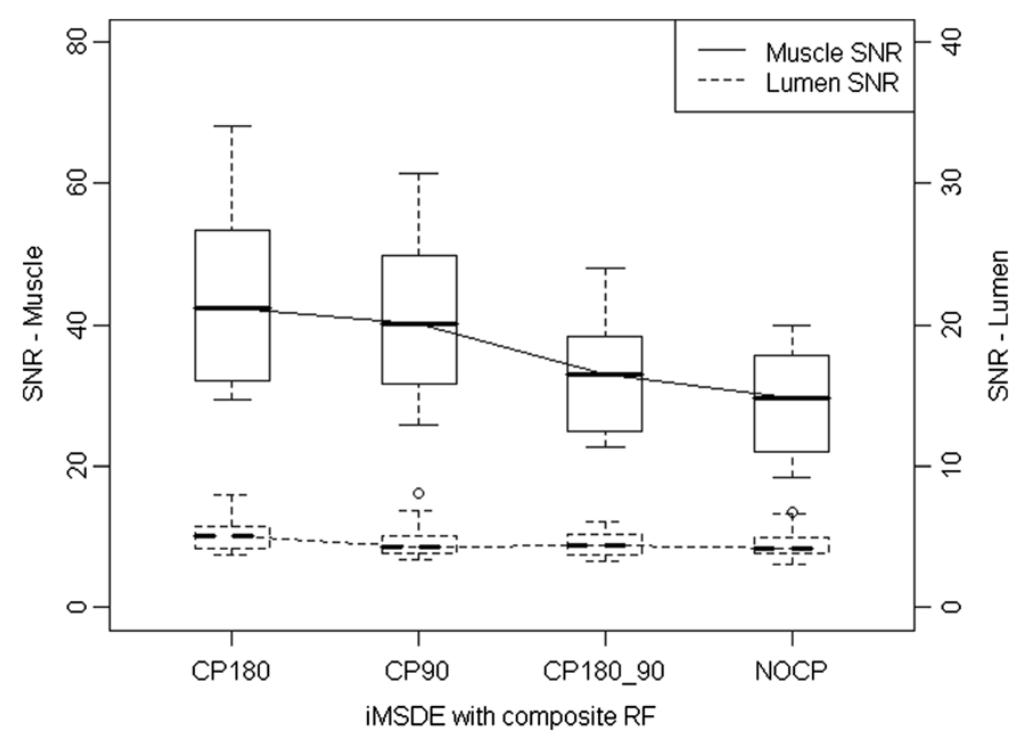

Figure 4 Comparison of muscle and lumen SNR with varying iMSDE RF pulse type in $\mathrm{T}_{1}$-w images (6 volunteers, 48 locations). Composite $180^{\circ}$ only (CP180), composite $-90^{\circ}$ only (CP90), composite $180^{\circ}$ and composite $-90^{\circ}$ (CP180_CP90), and no composite RF pulses (NOCP). Sinusoidal gradients with a first order moment of $487 \mathrm{mT}^{* *} \mathrm{~ms}^{2} / \mathrm{m}$ were used.

weightings used for plaque imaging. Furthermore, the choice of an optimal first order moment $\left(\mathrm{m}_{1}\right)$ within the iMSDE module is important, since $\mathrm{m}_{1}$ directly determines the blood suppression performance, and alters the image contrast and SNR.

In this study we demonstrated that a small $\mathrm{m}_{1}$ (487 $\mathrm{mT}^{*} \mathrm{~ms}^{2} / \mathrm{m}$ ) can achieve better blood suppression, albeit with reduced SNR in $\mathrm{T}_{1}$-w and $\mathrm{T}_{2}$-w FSE compared with conventional DIR preparation. We reported optimized $\mathrm{m}_{1}$ values to achieve blood suppression and SNR performance in both $\mathrm{T}_{1^{-}}$and $\mathrm{T}_{2}-\mathrm{w}$ FSE sequences. In theory, blood suppression of iMSDE improves with increasing $\mathrm{m}_{1}$. However, a large $m_{1}$ requires a longer preparation time that results in increased $T_{2}$ decay and further signal loss due to the greater eddy current effects, hence causes an overall decrease in SNR. Therefore, for $\mathrm{T}_{1} \mathrm{w}$ contrast, $\mathrm{m}_{1}$ needs to be minimized in order to reduce $T_{2}$ weighting and SNR loss. We found a small $\mathrm{m}_{1}\left(487 \mathrm{mT}^{*} \mathrm{~ms}^{2} / \mathrm{m}\right)$ can achieve satisfactory blood suppression, and at the same time minimize the SNR loss and $\mathrm{T}_{2}$ weighting (increasing the effective echo time by $13.6 \mathrm{~ms}$ ) (Figures 6 and 7). Nonetheless, the considerable SNR loss $(\sim 40 \%)$ in $\mathrm{T}_{1} \mathrm{~W}$ imaging can possibly limit its application in highresolution imaging when SNR is low. When SNR is adequate, iMSDE can be used in $\mathrm{T}_{1} \mathrm{w}$ imaging to reduce plaque mimicking flow artefacts. Other novel blood suppression techniques, such as Delayed Alternating with Nutation for Tailored Excitation (DANTE), which induces smaller $T_{2} / T_{1}$ contrast changes with less SNR loss, may be optimal for $\mathrm{T}_{1} \mathrm{w}$ imaging [19]. Recently, Li et al. found DANTE has a higher vessel wall CNR efficiency than iMSDE and DIR methods in multi-contrast FSE imaging [20].

We found iMSDE preparation can achieve better blood suppression and better/comparable muscle SNR than DIR in $\mathrm{T}_{2}-\mathrm{w}$ imaging without affecting image contrast by matching the effective echo times with $\mathrm{m}_{1}<=1518$ $\mathrm{mT}^{* *} \mathrm{~ms}^{2} / \mathrm{m}$ (Figures 6 and 7). Higher $\mathrm{m}_{1}$ values are not recommended as they lead to significant SNR loss without further improving the blood suppression.

Table 2 SNR and CNReff comparison of the permutations of composite RF pulse types (six volunteers)

\begin{tabular}{|c|c|c|c|c|c|c|}
\hline & Muscle SNR median [IQ] & p-value & Lumen SNR median [IQ] & p-value & CNReff median [IQ] & p-value \\
\hline \multicolumn{7}{|l|}{ iMSDE } \\
\hline CP180 & $50.2[11.4]$ & \multirow{4}{*}{0.151} & $5.4[1.4]$ & \multirow{4}{*}{0.387} & $20.9[4.7]$ & \multirow{4}{*}{0.154} \\
\hline CP180_90 & $44.7[11.7]$ & & $4.9[1.6]$ & & $19.2[3.8]$ & \\
\hline CP90 & $46.4[9.7]$ & & $5.2[1.5]$ & & $18.6[4.8]$ & \\
\hline NOCP & $47.7[12.6]$ & & $5.2[1.4]$ & & 19.9 [5.3] & \\
\hline
\end{tabular}

iMSDE preparations were performed using a sinusoid gradient with a $487 \mathrm{mT}^{*} \mathrm{~ms}^{2} / \mathrm{m}$ first order moment. 


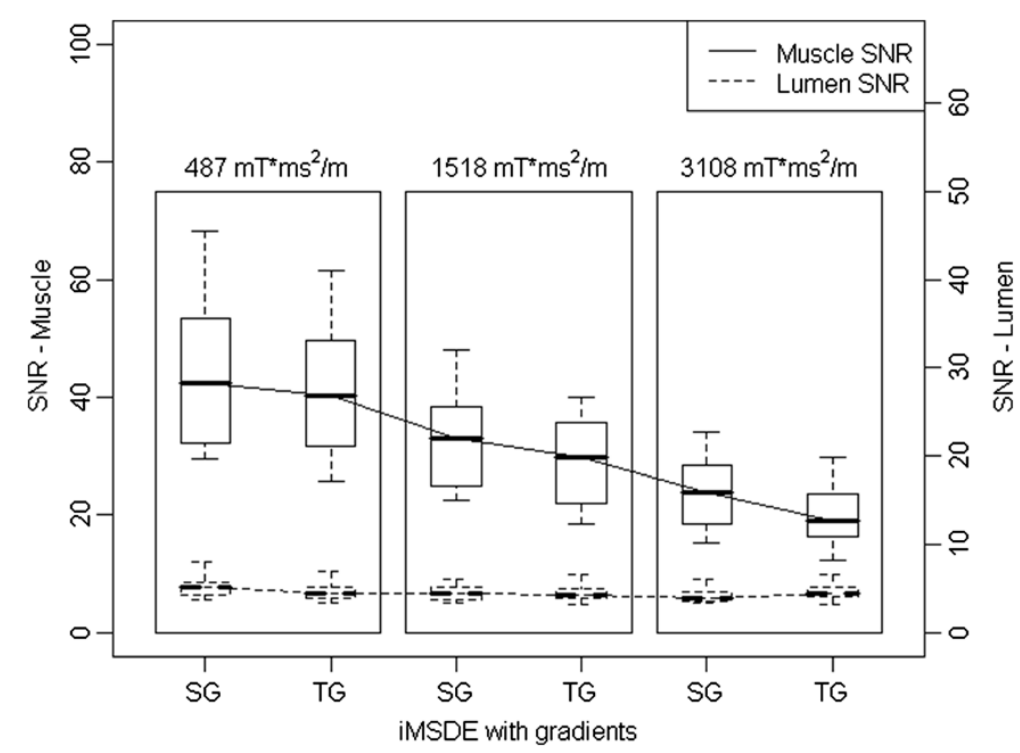

Figure 5 Comparison of muscle SNR change using iMSDE preparation with sinusoidal (SG) and trapezoidal (TG) gradients (6 volunteers, 48 locations).

As shown in the phantom study (Figure 3), plaque components with shorter $T_{2}$ values than muscle would be expected to have even greater SNR loss due to the iMSDE preparation time. This hypothesis was evaluated in the patient sub-study.

In the volunteer study we found that the iMSDE preparation using a composite $180^{\circ} \mathrm{RF}$ pulse reduced the signal loss due to $\mathrm{B}_{1}^{+}$non-uniformity (Figure 4), which agreed with the test object results (Figure 2), but no significant differences were found (Table 2). Composite $-90^{\circ}$ did not appear to improve the image uniformity (Figure 2) and also resulted in a slight SNR decrease (Figure 4). This was due to the composite $-90^{\circ}$ slightly increasing the iMSDE preparation time by $2.4 \mathrm{~ms}$ (the composite $-90^{\circ}$ pulse comprises a $-360^{\circ}$ pulse and a $270^{\circ}$ pulse with a total duration of
$2.8 \mathrm{~ms}$, while a simple $-90^{\circ}$ pulse has a duration of $0.4 \mathrm{~ms})$.

The use of sinusoidal flow dephasing gradients reduced the signal loss presumed to be caused by eddy current effects in both the phantom and volunteer data. This trend became more obvious at higher $\mathrm{m}_{1}$ (Figure 5 and Table 2), possibly due to the increased eddy currents when larger flow-sensitizing gradients were applied. An intuitive advantage of applying sinusoidal gradients is that the $\mathrm{dB} / \mathrm{dt}$ is relatively low which we would expect to lead to a reduction in eddy current induced artifacts [21].

Optimisation of arterial wall imaging using iMSDE blood suppression has potential clinical value, particularly for assessment of atheromatous plaques in the carotid artery. The carotid bifurcation is the most common anatomical site of atheroma formation. Due to irregular geometry and

Table 3 SNR and CNReff comparison of sinusoidal and trapezoidal motion sensitizing gradient with increased first order moment (six volunteers)

\begin{tabular}{|c|c|c|c|c|c|c|}
\hline & Muscle SNR median [IQ] & p-value & Lumen SNR median [IQ] & $\mathrm{p}$-value & Muscle CNReff median [IQ] & p-value \\
\hline \multicolumn{7}{|l|}{ iMSDE } \\
\hline \multicolumn{7}{|l|}{ Sinusoidal } \\
\hline $487 \mathrm{mT}^{*} \mathrm{~ms}^{2} / \mathrm{m}$ & $50.2[11.4]$ & & $5.4[1.4]$ & & $20.9[4.7]$ & \\
\hline $1518 \mathrm{mT}^{*} \mathrm{~ms}^{2} / \mathrm{m}$ & $37.1[8.1]$ & & $4.4[1.1]$ & & $15.3[3.8]$ & \\
\hline $3108 \mathrm{mT}^{*} \mathrm{~ms}^{2} / \mathrm{m}$ & $26.2[8.1]$ & & $4.2[1.4]$ & & $13.4[3.4]$ & \\
\hline Trapezoidal & & $<0.001^{*}$ & & 0.338 & & $<0.001^{*}$ \\
\hline $487 \mathrm{mT}^{*} \mathrm{~ms}^{2} / \mathrm{m}$ & $48.8[11.7]$ & & $4.8[1.4]$ & & $20.5[5.5]$ & \\
\hline $1518 \mathrm{mT}^{*} \mathrm{~ms}^{2} / \mathrm{m}$ & $33.1[7.3]$ & & $4.4[1.1]$ & & $14.2[4.9]$ & \\
\hline $3108 \mathrm{mT}^{*} \mathrm{~ms}^{2} / \mathrm{m}$ & $21.9[8.1]$ & & $4.7[1.2]$ & & $10.3[2.9]$ & \\
\hline
\end{tabular}

*: significant difference. 


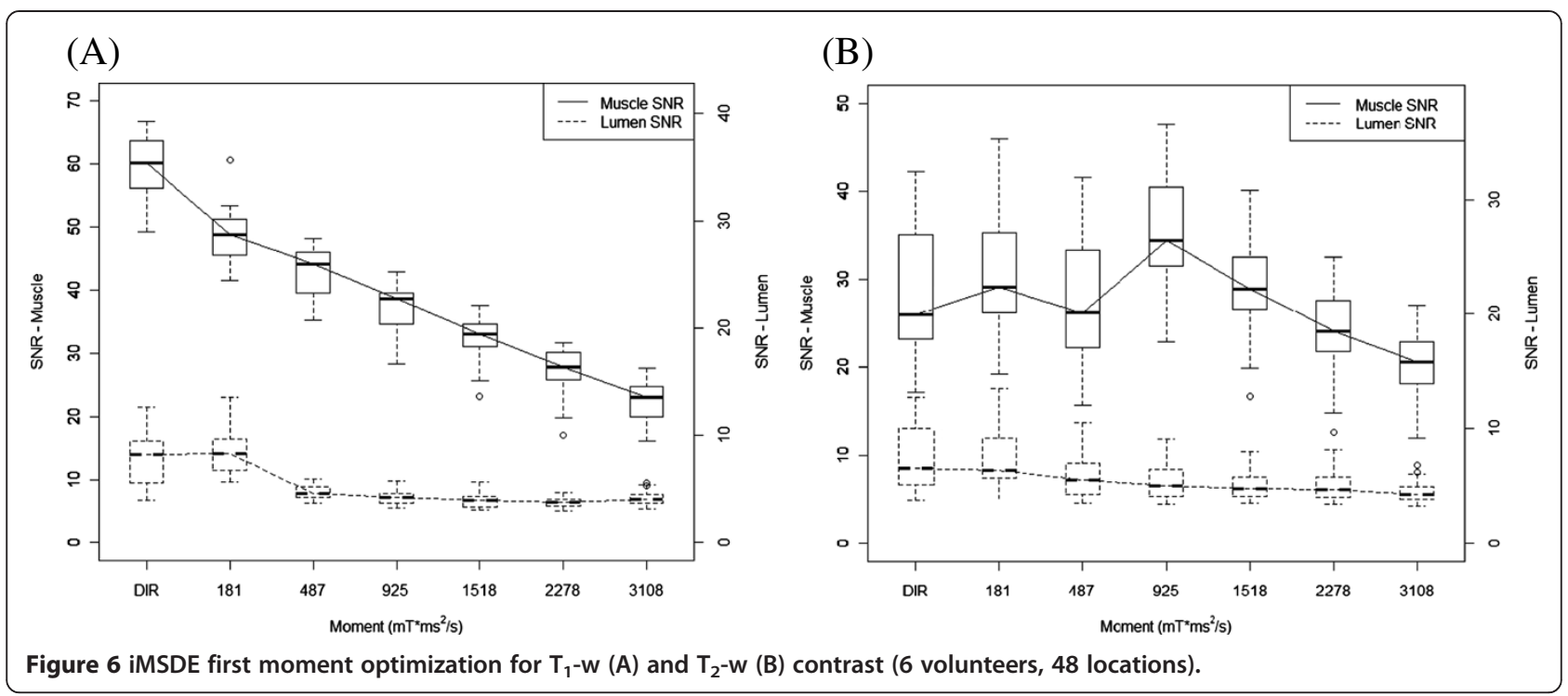

complex flow patterns particularly at higher grades of luminal stenosis, blood suppression can prove particularly challenging leading to plaque mimicking artefacts [3]. This may result in inaccurate measurements of plaque burden and luminal stenosis, both of which are clinically important criteria for refined severity assessment of carotid artery disease [22-24].

Plaque mimicking artefacts were frequently observed in our normal volunteer cohort (Figure 7). This may have been due to stagnant blood, which is common in subjects without luminal stenosis. This finding is also common in patients where medications such as $ß$-blockers may result in significant bradycardia. In our patient scans, blood flow artefacts were evident on both the $\mathrm{T}_{1} \mathrm{w}$ and $\mathrm{T}_{2} \mathrm{~W}$ imaging, and the optimized iMSDE module successfully suppressed the flow artefacts without degrading the image quality (Figure 8).

Compared to DIR, iMSDE was found to provide a substantial improvement in blood suppression, thereby distinguishing the arterial lumen from the inner wall boundary and minimizing the likelihood of mistaking flow artefacts for wall thickening (Figures 7 and 8). Moreover, compared

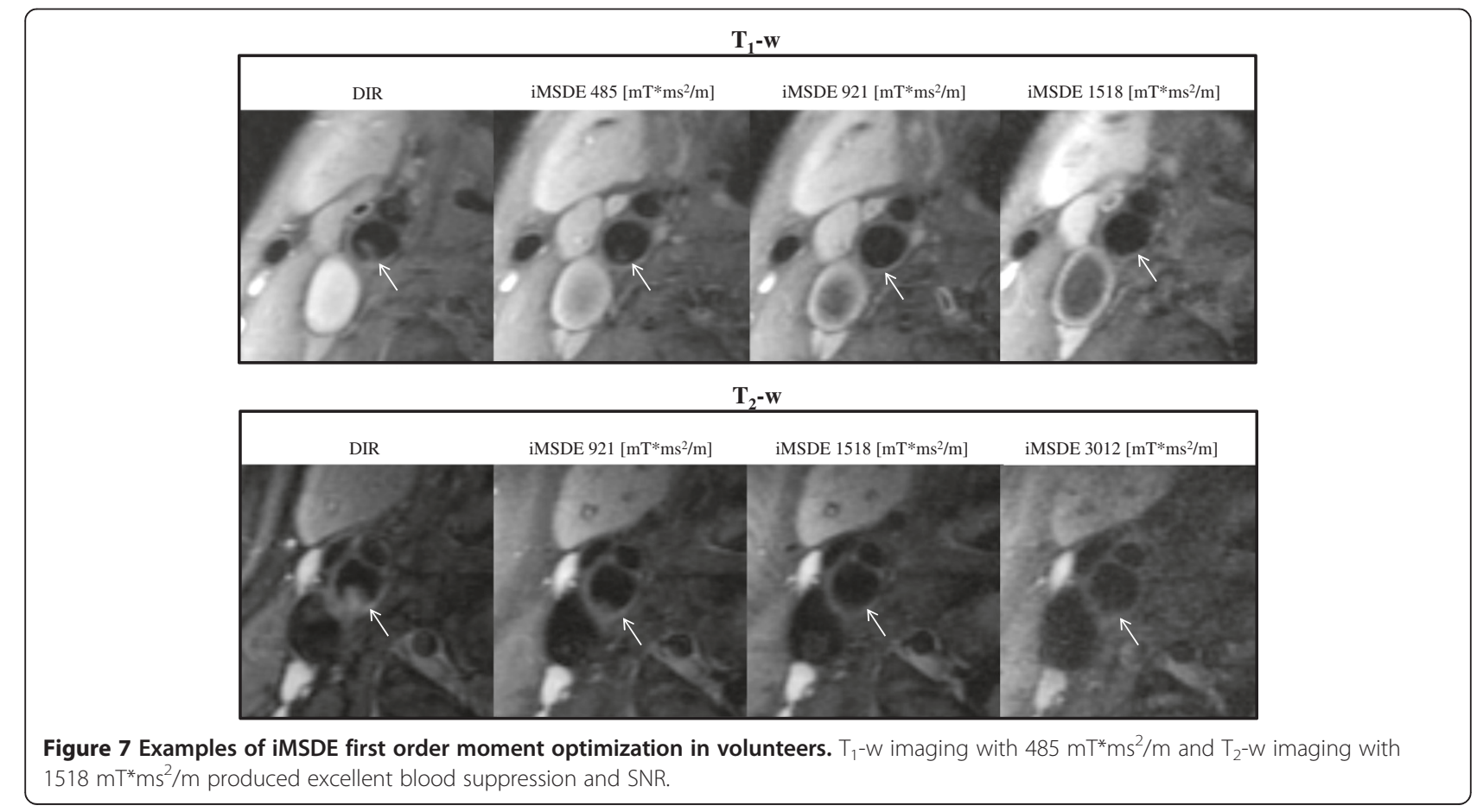




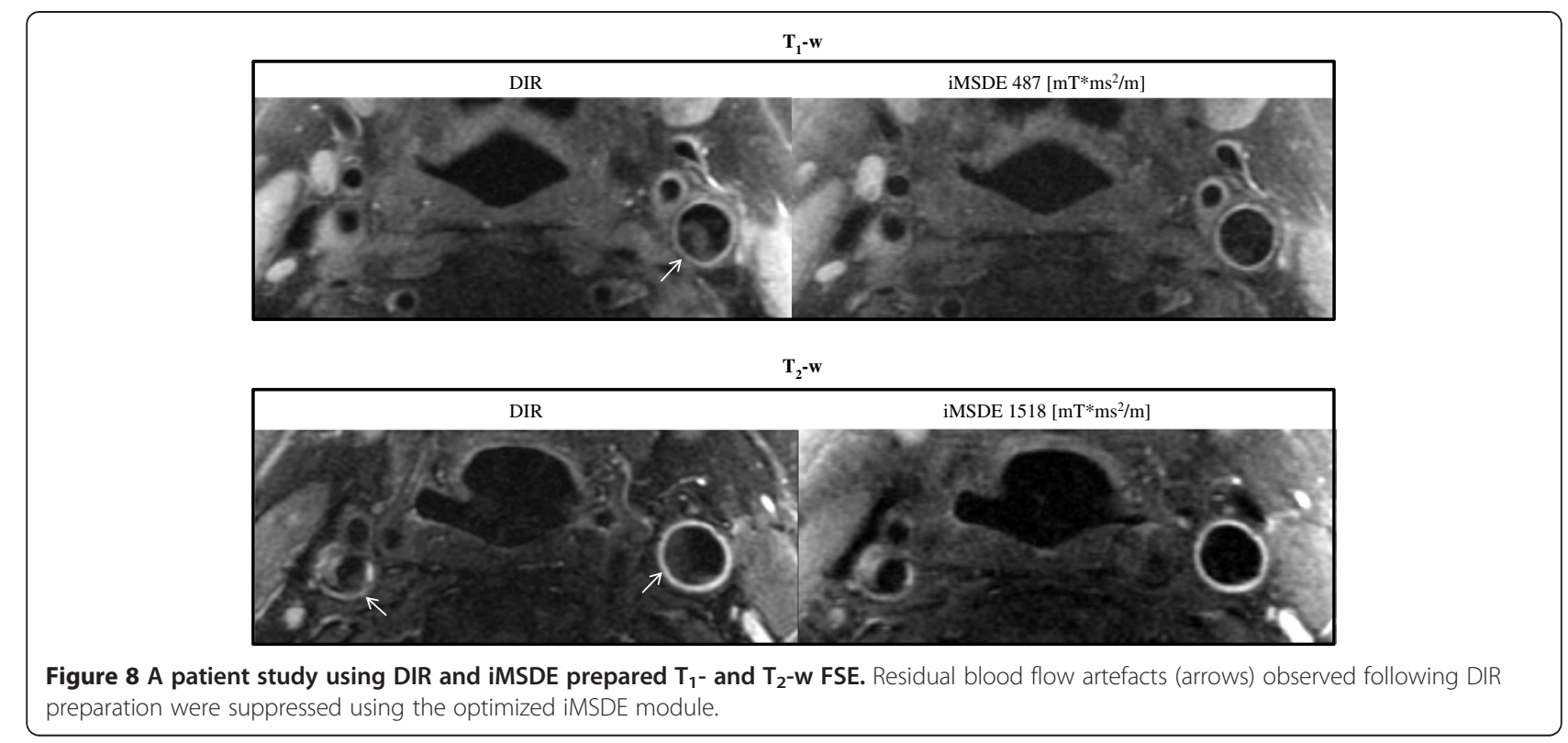

to trapezoidal flow dephasing gradients, the use of sinusoidal gradients resulted in an increase in muscle SNR (Figure 4) without compromising blood suppression. Also noteworthy is the fact that compared to DIR, iMSDE has a shorter preparation time $(\sim 20 \mathrm{~ms})$ compared with DIR (>200 ms) [4]. The time efficiency of iMSDE preparation makes this technique more useful for vessel wall imaging than DIR preparation in multislice imaging.

Motion sensitivity of iMSDE may cause carotid wall signal loss due to pulsatility. However, the pulsatile motion of the carotid vessel wall during the cardiac cycle (around $1 \mathrm{~s}$ ) is of the order of $0.4 \mathrm{~mm}$ [25], while the majority of the intra-luminal blood flow velocity is $\sim 10 \mathrm{~cm} / \mathrm{s}$ [26]. In the patient scan, we observed that in $\mathrm{T}_{1} \mathrm{w}$ imaging (using a small $\mathrm{m}_{1}$ of $487 \mathrm{mT}^{*} \mathrm{~ms}^{2} / \mathrm{m}$ ), the percentage wall SNR decrease was less than the muscle SNR reduction (Table 4); whilst in $\mathrm{T}_{2} \mathrm{~W}$ imaging (using a larger $\mathrm{m}_{1}$ of 1518 $\mathrm{mT}^{*} \mathrm{~ms}^{2} / \mathrm{m}$ ), the percentage wall SNR reduction was greater than the muscle SNR decrease. This is possibly due to the high $m_{1}$ inducing signal loss in the wall due to pulsatile motion. However, even with a high $\mathrm{m}_{1}$, the signal loss in the vessel wall was still considered acceptable $(\sim 20 \%)$ and no obvious image quality degradation was observed.

In this study iMSDE was compared relative to the ungated multi-slice DIR preparation as proposed by Yarnykh and Yuan as the de facto standard [4]. Subsequent work has demonstrated that the ungated approach produces comparable SNR and plaque measurements whilst reducing scan time [27]. It is also important to consider that gated sequences will often produce variable image contrast as a result of variability in triggering.

3D variable flip angle FSE imaging of carotid plaque is gaining popularity for its intrinsic black blood effect, high isotropic resolution, large coverage and high SNR efficiency [11-13]. However in some situations of complex flow, it can still exhibit residual flow artefacts [14].

Previously MSDE has been used with a $\mathrm{T}_{2} \mathrm{w}$ SPACE (Siemens Healthcare, equivalent to CUBE) acquisition to

Table 4 Comparison of muscle and wall SNR/CNReff in patients using optimized iMSDE and DIR (six patients)

\begin{tabular}{|c|c|c|c|c|c|}
\hline & $\begin{array}{l}\text { Muscle SNR } \\
\text { median [IQ] }\end{array}$ & $\begin{array}{l}\text { Wall SNR } \\
\text { median [IQ] }\end{array}$ & $\begin{array}{l}\text { Lumen SNR } \\
\text { median [IQ] }\end{array}$ & $\begin{array}{l}\text { Muscle CNR } \\
\text { median }[\mathrm{IQ}]\end{array}$ & $\begin{array}{l}\text { Wall CNR } \text { eff } \\
\text { median [IQ] }\end{array}$ \\
\hline \multicolumn{6}{|l|}{$\mathrm{T}_{1} \mathrm{~W}$} \\
\hline DIR & $62.8[17.3]$ & 31.8 [10.0] & $9.4[5.0]$ & $24.9[5.7]$ & $9.5[6.3]$ \\
\hline iMSDE $487 \mathrm{mT}^{*} \mathrm{~ms}^{2} / \mathrm{m}$ & $37.5[9.0]$ & $20.8[8.8]$ & $5.4[4.2]$ & $15.0[2.3]$ & $6.5[4.0]$ \\
\hline$p$ value & $<0.001^{*}$ & $<0.001^{*}$ & $0.02^{*}$ & $<0.001^{*}$ & $<0.001^{*}$ \\
\hline \multicolumn{6}{|l|}{$\mathrm{T}_{2} \mathrm{~W}$} \\
\hline $\mathrm{DIR}$ & $26.0[7.8]$ & $19.8[11.5]$ & $6.2[3.1]$ & $9.3[2.2]$ & $6.4[4.8]$ \\
\hline iMSDE $1518 \mathrm{mT}^{*} \mathrm{~ms}^{2} / \mathrm{m}$ & $27.5[6.5]$ & 15.4 [10.5] & $5.3[1.7]$ & $10.4[2.2]$ & $4.7[5.0]$ \\
\hline$p$ value & $<0.001^{*}$ & $0.01^{*}$ & $<0.001^{*}$ & $<0.001^{*}$ & $<0.001^{*}$ \\
\hline
\end{tabular}

\footnotetext{
*: significant difference.
} 

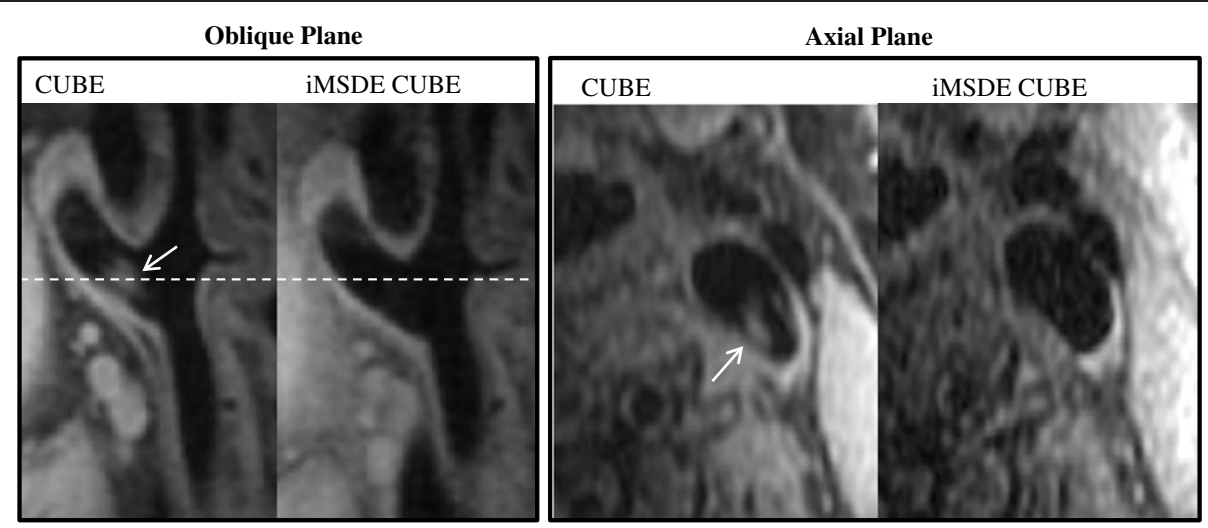

Figure 9 Example images of 3D $\mathrm{T}_{1}$-w CUBE and iMSDE prepared CUBE images in a volunteer. Blood flow artefacts (arrows) were further suppressed with the iMSDE preparation, however, a reduction in SNR was noted.

improve the blood suppression [14]. Similarly, we have shown that our optimized iMSDE module can also improve the blood suppression in $\mathrm{T}_{1} \mathrm{w}$ CUBE. However, we did note a reduction in SNR. This was possibly a result of the shorter TR ( $440 \mathrm{~ms}$ ) used in our $\mathrm{T}_{1} \mathrm{w}$ CUBE implementation. Since 3D FSE acquisitions such as CUBE and SPACE have an intrinsic black blood effect, a smaller $\mathrm{m}_{1}$ maybe sufficient for additional blood suppression if necessary. Future work will systematically investigate optimal blood suppression regimes appropriate for 3D CUBE.

\section{Conclusions}

iMSDE with small $\mathrm{m}_{1}\left(487 \mathrm{mT}^{*} \mathrm{~ms}^{2} / \mathrm{m}\right)$ achieves better blood suppression but reduced vessel wall CNR efficiency in $\mathrm{T}_{1^{-}}$and $\mathrm{T}_{2}$-w images relative to conventional DIR preparations. iMSDE is promising for $\mathrm{T}_{2-\mathrm{W}}$ imaging, however, we noted that it significantly reduced SNR and induced extra $\mathrm{T}_{2}$ weightings when applied in $\mathrm{T}_{1}$-w imaging. Optimized $\mathrm{m}_{1}$ for $\mathrm{T}_{1}-\mathrm{w}\left(487 \mathrm{mT}^{*} \mathrm{~ms}^{2} / \mathrm{m}\right)$ and $\mathrm{T}_{2}$ - $\mathrm{w}(1518$ $\mathrm{mT}^{*} \mathrm{~ms}^{2} / \mathrm{m}$ ) FSE are determined. The use of composite $180^{\circ}$ refocusing pulses and sinusoidal gradients improves the SNR performance without compromising blood suppression efficiency. iMSDE is also suitable for 3D CUBE acquisitions and can further improve its inherent blood suppression.

\section{Abbreviations}

iMSDE: Improved motion-sensitized driven-equilibrium; SNR: Signal to noise ratio; DIR: Double inversion recovery; FSE: Fast spin echo; RF: Radiofrequency; NEX: Number of excitations; ETL: Echo train length; FOV: Field of view: $\mathrm{T}_{1}$-w: $\mathrm{T}_{1}$-weighted; $\mathrm{T}_{2}-\mathrm{w}: \mathrm{T}_{2}$-weighted; PD-w: Proton-density-weighted.
}

\section{Competing interests}

The authors declare that they have no competing interests.

\section{Authors' contributions}

CZ designed the study, developed the MR sequences, processed the data and wrote the manuscript; MJG developed the MR sequences and revised the manuscript; JY undertook image analysis tasks and revised the manuscript; US recruited the volunteers and patients and revised the manuscript; JHG processed the data and revised the manuscript; AJP designed the study, processed the data and revised the manuscript. All authors read and approved the manuscript.

\section{Acknowledgements}

The project was supported by the Addenbrooke's Charitable Trust and the NIHR comprehensive Biomedical Research Centre award to Cambridge University Hospitals NHS Foundation Trust in partnership with the University of Cambridge, and partly supported by ARTreat European Union Frame Project 7.

\section{Author details}

${ }^{1}$ University Department of Radiology, University of Cambridge, Cambridge CB2 OQQ, UK. ${ }^{2}$ Cambridge Vascular Unit, Cambridge University Hospitals NHS Foundation Trust, Cambridge CB2 OQQ, UK.

Received: 25 July 2013 Accepted: 28 July 2014 Published: 9 August 2014

\section{References}

1. Lusis AJ. Atherosclerosis. Nature. 2000; 407:233-41.

2. Underhill HR, Hatsukami TS, Fayad ZA, Fuster V, Yuan C. MRI of carotid atherosclerosis: clinical implications and future directions. Nat Rev Cardiol. 2010; 7:165-73.

3. Steinman DA, Rutt BK. On the nature and reduction of plaque-mimicking flow artifacts in black blood MRI of the carotid bifurcation. Magn Reson Med. 1998; 39:635-41.

4. Yarnykh VL, Yuan C. Multislice double inversion-recovery black-blood imaging with simultaneous slice reinversion. J Magn Reson Imaging. 2003; 17:478-83.

5. Wang J, Yarnykh VL, Hatsukami T, Chu B, Balu N, Yuan C. Improved suppression of plaque-mimicking artifacts in black-blood carotid atherosclerosis imaging using a multislice motion-sensitized driven-equilibrium (MSDE) turbo spin-echo (TSE) sequence. Magn Reson Med. 2007; 58:973-81.

6. Koktzoglou I, Li D. Diffusion-prepared segmented steady-state free precession: application to 3D black-blood cardiovascular magnetic resonance of the thoracic aorta and carotid artery walls. J Cardiovasc Magn Reson. 2007; 9:33-42.

7. Balu N, Yarnykh VL, Chu B, Wang J, Hatsukami T, Yuan C. Carotid plaque assessment using fast 3D isotropic resolution black-blood MRI. Magn Reson Med. 2011; 65:627-37.

8. Wang J, Yarnykh VL, Yuan C. Enhanced image quality in black-blood MRI using the improved motion-sensitized driven-equilibrium (iMSDE) sequence. J Magn Reson Imaging. 2010; 31:1256-63.

9. Toussaint JF, LaMuraglia GM, Southern JF, Fuster V, Kantor HL. Magnetic resonance images lipid, fibrous, calcified, hemorrhagic, and thrombotic components of human atherosclerosis in vivo. Circulation. 1996; 94:932-38

10. Brittain JH, Hu BS, Wright GA, Meyer CH, Macovski A, Nishimura DG. Coronary angiography with magnetization-prepared T2 contrast. Magn Reson Med. 1995; 33:689-96.

11. Jara H, Yu BC, Caruthers SD, Melhem ER, Yucel EK. Voxel sensitivity function description of flow-induced signal loss in MR imaging: implications for 
black-blood MR angiography with turbo spin-echo sequences. Magn Reson Med. 1999; 41:575-90.

12. Mihai G, Winner MW, Raman SV, Rajagopalan S, Simonetti OP, Chung YC. Assessment of carotid stenosis using three-dimensional T2-weighted dark blood imaging: Initial experience. J Magn Reson Imaging. 2012; 35:449-55.

13. Takano K, Yamashita S, Takemoto K, Inoue T, Sakata N, Kuwabara Y, Yoshimitsu K. Characterization of carotid atherosclerosis with black-blood carotid plaque imaging using variable flip-angle 3D turbo spinecho: comparison with 2D turbo spin-echo sequences. Eur J Radiol. 2011; 81:e304-09.

14. Fan Z, Zhang Z, Chung YC, Weale P, Zuehlsdorff S, Carr J, Li D. Carotid arterial wall MRI at $3 \mathrm{~T}$ using $3 \mathrm{D}$ variable-flip-angle turbo spin-echo (TSE) with flow-sensitive dephasing (FSD). J Magn Reson Imaging. 2010; 31:645-54.

15. Mavrogeni S, Tzelepis GE, Athanasopoulos G, Maounis T, Douskou M Papavasiliou A, Cokkinos DV. Cardiac and sternocleidomastoid muscle involvement in Duchenne muscular dystrophy: an MRI study. Chest. 2005; 127:143-48

16. Biasiolli L, Lindsay AC, Chai JT, Choudhury RP, Robson MD. In-vivo quantitative T2 mapping of carotid arteries in atherosclerotic patients: segmentation and T2 measurement of plaque components. J Cardiovasc Magn Reson. 2013; 15:69.

17. Constantinides $C D$, Atalar E, McVeigh ER. Signal-to-noise measurements in magnitude images from NMR phased arrays. Magn Reson Med. 1997; 38:852-57.

18. Obara M, VAN Cauteren M, Honda M, Imai Y, Kuroda K. Assessment of Improved Motion-Sensitized Driven Equilibrium (iMSDE) for multi-contrast vessel wall screening. Magn Reson Med Sci. 2014; 13:139-44.

19. Li L, Miller KL, Jezzard P. DANTE-prepared pulse trains: a novel approach to motion-sensitized and motion-suppressed quantitative magnetic resonance imaging. Magn Reson Med. 2012; 68:1423-38.

20. Li L, Chai JT, Biasiolli L, Robson MD, Choudhury RP, Handa Al, Near J, Jezzard P. Black-Blood Multicontrast Imaging of Carotid Arteries with DANTEprepared 2D and 3D MR Imaging. Radiology. 2014; 131717.

21. Derek K. Diffusion MRI: Theory, methods, and applications. Oxford University Press; 2011.

22. European MRC. Carotid surgery trial: interim results for symptomatic patients with severe $(70-99 \%)$ or with mild $(0-29 \%)$ carotid stenosis. European Carotid Surgery Trialists' Collaborative Group. Lancet. 1991; 337:1235-43.

23. North American Symptomatic Carotid Endarterectomy Trial Collaborators. Beneficial effect of carotid endarterectomy in symptomatic patients with high-grade carotid stenosis. North American Symptomatic Carotid Endarterectomy Trial Collaborators. N Engl J Med. 1991; 325:445-53.

24. Bots ML, Hoes AW, Koudstaal PJ, Hofman A, Grobbee DE. Common carotid intima-media thickness and risk of stroke and myocardial infarction: the rotterdam study. Circulation. 1997; 96:1432-37.

25. Selzer RH, Mack WJ, Lee PL, Kwong-Fu H, Hodis HN. Improved common carotid elasticity and intima-media thickness measurements from computer analysis of sequential ultrasound frames. Atherosclerosis. 2001; 154:185-93.

26. Lal BK, Hobson RW 2nd, Tofighi B, Kapadia I, Cuadra S, Jamil Z. Duplex ultrasound velocity criteria for the stented carotid artery. J Vasc Surg. 2008; 47:63-73.

27. Mani V, Itskovich W, Aguiar SH, Mizsei G, Aguinaldo JG, Samber DD, Macaluso FM, Fayad ZA. Comparison of gated and non-gated fast multislice black-blood carotid imaging using rapid extended coverage and inflow/outflow saturation techniques. J Magn Reson Imaging. 2005; 22:628-33.

doi:10.1186/s12968-014-0061-5

Cite this article as: Zhu et al:: Optimization of Improved Motion-sensitized Driven-equilibrium (iMSDE) blood suppression for carotid artery wall imaging. Journal of Cardiovascular Magnetic Resonance 2014 16:61.

\section{Submit your next manuscript to BioMed Central and take full advantage of:}

- Convenient online submission

- Thorough peer review

- No space constraints or color figure charges

- Immediate publication on acceptance

- Inclusion in PubMed, CAS, Scopus and Google Scholar

- Research which is freely available for redistribution

Submit your manuscript at www.biomedcentral.com/submit
() Biomed Central 